\title{
Transposition
}

\section{La musique comme voie possible d'une histoire comparée des conflits armés.}

Entretien avec Didier Francfort

A comparative history of armed conflicts through music. Interview with Didier

Francfort

\section{Luis Velasco-Pufleau}

\section{OpenEdition}

\section{Journals}

Édition électronique

URL : http://journals.openedition.org/transposition/512

DOI : 10.4000/transposition. 512

ISSN : $2110-6134$

Éditeur

CRAL - Centre de recherche sur les arts et le langage

\section{Référence électronique}

Luis Velasco-Pufleau, « La musique comme voie possible d'une histoire comparée des conflits armés. », Transposition [En ligne], 4 | 2014, mis en ligne le 15 juillet 2014, consulté le 19 avril 2019. URL: http://journals.openedition.org/transposition/512; DOI : 10.4000/transposition.512

Ce document a été généré automatiquement le 19 avril 2019.

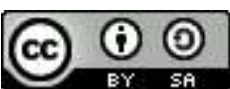

La revue Transposition est mise à disposition selon les termes de la Licence Creative Commons Attribution - Partage dans les Mêmes Conditions 4.0 International. 


\title{
La musique comme voie possible d'une histoire comparée des conflits armés.
}

\author{
Entretien avec Didier Francfort \\ A comparative history of armed conflicts through music. Interview with Didier \\ Francfort
}

Luis Velasco-Pufleau

\section{Une approche historique comparée de la musique}

LUIS Velasco-pufleau - Pourriez-vous nous expliquer comment, dans votre carrière d'historien, vous en êtes venu à travailler sur la musique?

Didier Francfort - Je suis arrivé à travailler sur la musique par paresse, tout simplement. En fait, j'ai fait une thèse sur l'histoire de la sociabilité et la vie associative dans le nord de l'Italie, dans le Frioul - dans la ville d'Udine - au moment où la région passait de l'empire des Habsbourg au Royaume d'Italie ${ }^{1}$. A cette époque, j'ai fait un travail historique classique en dépouillant la presse, des archives d'associations et des sociétés - des clubs sportifs, des sociétés agraires - et aussi la liste des abonnés au théâtre. Finalement cela a été beaucoup de travail, beaucoup d'efforts pour en définitive peu de résultats.

Alors qu'en même temps, je voyais que la programmation de l'opéra, qui s'appelait Théâtre Social, permettait de percevoir peut-être mieux et beaucoup plus vite les réels changements dans les représentations et dans les mécanismes sociaux. Donc, en reprenant la recherche après la soutenance de ma thèse, je me suis dit que c'était peutêtre par ce biais que j'arriverais à mieux comprendre, et de façon plus rapide, ces phénomènes de changements historiques et de mutations sociales.

Cela m'a semblé une sorte de raccourci, et c'est pour ça que j'ai dit que c'est par paresse que j'en suis arrivé là, mais c'est aussi pour une autre raison - sinon je vais apparaître comme quelqu'un d'épouvantablement paresseux. L'autre raison est que je suis 
persuadé que pour comprendre les phénomènes sociaux, il faut sortir du cadre national et faire une histoire comparée. D'abord parce qu'il y a beaucoup de réalités transnationales mais aussi parce que par moments il est intéressant de voir ce qu'il peut y avoir de commun dans des évolutions sociales au sein de cadres nationaux différents. Or, cette démarche comparée nécessiterait de parler un tas de langues - en ce qui me concerne, je comprends à peu près le français, l'italien, l'anglais et un minimum d'allemand, pour le reste j'ai de toutes petites notions. Si nous nous en tenons à des sources écrites, soit nous faisons du binational étroit, soit nous considérons qu'il y a des nations centrales et des nations périphériques. Et cela est inacceptable d'un point de vue à la fois historique, scientifique mais aussi moral et politique. Ce n'est pas parce qu'on a le malheur d'être Estonien qu'on n'a pas d'histoire, ou qu'on n'a pas d'histoire européenne. Donc, pour ne pas exclure les Estoniens ou d'autres, il m'a semblé que la musique est l'une des voies possibles pour faire une histoire européenne - à l'époque où j'ai entrepris ce travail nous ne parlions pas encore de globalisation ni d'histoire mondiale, on parlait seulement d'histoire européenne. Je ne crois pas donc qu'on puisse faire d'histoire européenne sans essayer, par la musique, d'accéder directement à des sources permettant de rendre compte des pratiques sociales de ces différents espaces qu'autrement nous percevrions comme périphériques. C'est pour cette raison qu'il me semble indispensable de le faire.

LvP - Sur le plan méthodologique, une fois que vous avez collecté ou analysé un nombre considérable de données ou d'exemples musicaux, comment les utilisez-vous ?

DF - Actuellement, j'ai tendance à accumuler et à voir comment ça marche, à voir comment cela interagit et se répond. Je vais prendre un exemple simple, qui est celui de voir comment les marches militaires sont perçues dans les différents espaces géographiques. Le concert de plein air qu'on allait voir en famille dans un parc est, si on lit du Zweig, typiquement viennois avant 1914, mais il est peut-être moins courant dans d'autres conditions sociales ou dans d'autres cadres nationaux. Il peut donc être intéressant de les comparer avec le fonctionnement d'autres marches, des marches militaires hongroises ou des marches militaires espagnoles, et pour cela nous pouvons parfois recourir à une démarche expérimentale. Par exemple, il y a un test très intéressant à réaliser dans les conférences, celui de voir si les gens tapent du pied à l'écoute d'une marche militaire. En effet, je passe deux marches et j'observe dans laquelle les gens tapent du pied et dans laquelle les gens ne tapent pas du pied, puis je me dis «voilà une efficacité sociale redoutable». Et ensuite je peux commencer à comparer les deux, à voir ce qui fait que certaines marches font que les gens tapent du pied et que d'autres restent dans les tiroirs de l'inutilité sociale, heureusement impossibles à récupérer par les pouvoirs.

Voilà une des voies possibles de l'utilisation, c'est-à-dire que l'avantage de travailler dans la jonction entre musique et histoire permet aussi un petit peu de démarche expérimentale qu'on aurait du mal à voir dans d'autres domaines historiques. Enfin, on espère que les gens qui travaillent sur les massacres et sur les guerres mondiales ne s'amusent pas à reproduire chez eux des conflits pour voir comment cela fonctionne. Voir comment fonctionne une musique sur un groupe social donné dans un moment déterminé, c'est un moindre mal.

Quoique... participer à ces moments d'unanimité dans les célébrations et les manifestations où tout le monde se lève et chante la même chose en même temps... Nous pouvons penser maintenant que cela est anodin, mais si nous avions été en 1935 à 
Nuremberg et que nous nous étions mis à chanter avec tout le monde... C'est pourquoi il est important de comprendre le mécanisme qui fait qu'on chante à un moment donné avec tout le monde, ce n'est pas si léger que ça et ce n'est pas anodin, il ne s'agit pas de faire une histoire uniquement pour le plaisir.

LVP - D'après vous, qu'est-ce que l'étude de la musique apporte à la discipline historique et aux sciences sociales?

DF - Il me semble évident que la musique permet de comparer, et que l'aspect comparatif élargit le champ des références. Mais aussi, l'étude de la musique permet de mettre en évidence des phénomènes d'imaginaires en partie collectifs que les textes ne permettent pas toujours d'appréhender. Il est clair pour moi que dans les hymnes nationaux, le rythme de l'hymne est un indicateur des représentations collectives de ce qui fait la nation. Quand nous prenons les hymnes nationaux qui «marchent» - la Marseillaise, Fratelli d'Italia - il y a quelque chose de l'ordre de la nation mobilisée. Dans les hymnes tristes, comme l'hymne national hongrois - «que Dieu protège les hongrois parce qu'ils ont assez souffert »- nous ne sommes pas dans le même type de mobilisation. Et quand nous arrivons à l'hymne de la minorité hongroise des Sicules de Transylvanie, c'est encore plus triste... Ou bien quand nous sommes dans le côté plaisant et mélodieux de l'hymne tchèque, il ne s'agit pas du même type de représentation. Donc l'hymne est un bon indicateur de ce qui caractérise la construction et la représentation symbolique de la nation, la façon de ressentir un imaginaire collectif construit.

\section{La musique au sein des conflits armés}

LVP - Dans certains de vos travaux, vous vous êtes intéressé à la fonction sociale des musiques militaires. Que pouvez-vous nous dire du rôle social de la musique dans les conflits armés européens, aussi bien dans l'accompagnement des combats que dans la mobilisation de la population civile?

DF - J'aurais tendance à dédouaner la musique de bien des responsabilités dans la «brutalisation » des sociétés en guerre. On a en définitive peu utilisé la musique dans les moments les plus violents. Il est difficile de parler de façon générale sans tenir compte de la spécificité de chaque conflit mais la même musique peut être utilisée de façon tout à fait contradictoire: Wagner ne donne pas toujours envie d' «envahir la Pologne ", comme le dit si plaisamment Woody Allen, même si depuis Apcalypse Now de Coppola (1979), on associe différemment des opérations guerrières à la musique de Wagner. Je m'intéresse beaucoup actuellement aux musiques populaires, pas nécessairement folkloriques ou "folklorisées", jouées, présentes dans les films de comédie musicale dans l'Union Soviétique stalinienne ou l'Allemagne nazie. On est loin d'un martèlement permanent de marches militaires. Il arrive même qu'avec Alexander Tsfasman ou Peter Igelhoff, cela swingue diablement. Ce n'est donc pas un simple reflet passif d'une société mobilisée par un régime totalitaire, ni un pousse-au-crime permanent. On ne saurait réduire le rôle de la musique dans les guerres aux marches militaires qui accompagnent les phases de mobilisation ou les défilés de victoire. Des chants élégiaques, des parodies, des reprises caractérisent les périodes de conflits autant que des formes d'instrumentalisation directe de la musique.

LvP - En temps de guerre, les œuvres musicales gardent donc des fonctions sociales multiples qui échapperaient - au moins en partie - au contrôle des institutions de l'État et 
permettraient leur investissement par des acteurs divers. Pourriez-vous nous en dire un peu plus sur le rôle de la musique « légère » au sein de l'Union Soviétique sous Staline et de l'Allemagne nazie dans le cas spécifique du deuxième conflit mondial?

DF - Question intéressante mais compliquée. Je vais essayer d'y répondre mais je ne suis pas sûr que cela apporte beaucoup de donner une réponse commune pour ces deux régimes totalitaires, mobilisant de façon constante la société et dépassant en violence toutes les vieilles dictatures. On a pu voir sur Arte un excellent documentaire réalisé, je crois en 2003, par Oliver Axer et Suzanne Benzel sur les « refrains du nazisme », le « hitparade» ou le «Schlager» d'Hitler. Le film est parfaitement réalisé, très impressionnant, il associe des images d'actualités, des scènes de cinéma, des films de propagande, des films d'amateurs avec un fond musical constitué par les grands succès de l'époque. C'est fait de façon brillante mais cela aboutit, à mon avis, à une forme de surinterprétation du rôle de la musique légère sous le régime nazi. La musique légère apparaît en effet uniquement dans son rôle de manipulation des masses en étant associée à des vues de ce qui se passe, y compris de témoignages de la violence exercée par le régime. Les refrains à la mode dans les démocraties en guerre étaient-ils si différents ? Le destin de la fameuse Lili Marleen est à cet égard emblématique. Le film réalisé en 1980 par Fassbinder retrace librement le singulier destin de la chanteuse qui a créé ce tube. Elle s'appelle Lale Andersen, c'est une représentante de la culture «maritime » de l'Allemagne du Nord. Elle a eu une liaison avec Rolf Liebermann (le futur directeur de l'Opéra de Paris). Pendant quelques années la chanson a été « un bide ". Puis après une diffusion par la radio allemande des troupes d'occupation à Belgrade, le succès est venu, les adaptations ont suivi et la chanson est passée dans l'autre camp, ne serait-ce que parce qu'elle a été reprise par Marlène Dietrich. Mais dans la France des débuts de la Guerre, le succès emblématique est J'attendrai, chanson créée en France en 1938, par la chanteuse italienne Rina Ketty, avec des paroles de Louis Poterat, sur une musique de Dino Olivieri qui avait déjà été un succès dans l'Italie mussolinienne, un peu comme une reprise « légère " du thème de Madame Butterfly. Mais la chanson acquiert, au début de la Guerre, un sens nouveau associé à l'attente des soldats partis au front puis des prisonniers de guerre. Je reviens à l'Allemagne et au film documentaire sur le hit-parade qui tient à démontrer que la volonté de distraire les foules a conduit à privilégier une esthétique «fleur bleue ", un peu niaise en tout cas, complètement coupée de toute influence des musiques étrangères, en particulier de la musique " dégénérée ». Certes, il y eut un nombre considérable de musiciens qui furent directement les victimes du nazisme et furent emprisonnés, assassinés ou exilés parce qu'ils étaient juifs, engagés politiquement à gauche, parfois les deux. Il s'agit parfois de compositeurs «savants» qui ne dédaignaient pas les musiques populaires, en particulier le jazz et le tango, Weill, Dessau, Eisler. La liste serait longue. Je pense en particulier aux chanteurs acteurs des films musicaux : Kurt Gerron, Josef Schmidt. Les hôtels berlinois à l'époque de la République de Weimar vibraient et dansaient en écoutant de fameux orchestres dirigés par des violonistes juifs issus d'Europe centrale et orientale comme Dajos Béla. Et puis il y a le prodigieux phénomène vocal du groupe des Comedian Harmonists. Le groupe dut se séparer et les chanteurs juifs s'exilèrent alors que les autres reprirent le groupe « aryanisé » avec la magnifique voix de basse de Robert Biberti et devint le Meistersextett. On continuait pendant la guerre à jouer la musique de Peter Kreuder qui s'était réfugié en Suède. Le discours officiel bannit tout ce qui évoque le jazz et l'Amérique des Noirs et des Juifs mais dans les pratiques musicales, bien des aspects du swing peuvent passer. J'ai parlé de Peter Igelhoff. Il n'est 
pas complètement isolé. La propagande nazie a même instrumentalisé le succès du swing en diffusant sur les ondes des succès et des standards américains repris, avec des paroles favorables au régime et méprisant Churchill et les Bolcheviks, par un Orchestre constitué d'après des directives de Goebbels, l'orchestre de Charlie, conduit par le chanteur Karl Schwedler avec le tromboniste Willy Berking et le batteur Fritz "Freddie" Brocksieper.

Du côté soviétique, la Grande Guerre Patriotique permet entre 1941 et 1944 une relative libéralisation dont bénéficient certains musiciens de jazz. Le trompettiste Eddie Rosner, d'origine juive polonaise, qui s'est réfugié en URSS, devient un musicien très populaire, connu comme le «Louis Armstrong blanc", alors qu'après la guerre il est interné au Goulag, victime du retour de la violence antisémite et de la volonté d'éradiquer l'influence culturelle américaine. La Seconde Guerre mondiale est ainsi associée à quelques chansons emblématiques. Ainsi la chanson composée en 1938 par le très prolifique Matveï Isaakovitch Blanter, Katioucha, tellement connue qu'elle passe pour être une vieille chanson populaire, est devenue un symbole. Son titre (le diminutif affectueux d'Ekaterina) est devenu le nom russe des lance-roquettes soviétiques que les Allemands appelaient «les orgues de Staline». Cette chanson a été reprise par la Résistance italienne, comme un hymne Fischia il vento, sur des paroles de Felice Cascione (mort en 1944). En France, la mélodie - qui n'était pas attribuée à Blanter servit pour lancer en 1969 le Casatchok. Une valse évoquant un foulard bleu, et le retour de l'absent, est devenue un autre symbole de la guerre, en particulier dans la version de Klavdiya Shulzhenko. En fait, il s'agissait avant la guerre d'un succès populaire polonais composé par Jerzy Peterburski. Ces chansons sont devenues des formes significatives de la mobilisation soviétique, en forçant le trait je dirais au moins autant que les symphonies de Chostakovitch.

LVP - Revenons un moment à Wagner et aux utilisations contradictoires des œuvres musicales dans différents contextes de représentation. Pensez-vous que la ritualisation de la musique suffise pour conférer aux œuvres des significations différentes de celles voulues par les compositeurs et par les contextes historiques de leur création ? Y aurait-il des spécificités rituelles propres aux sociétés en guerre?

DF - La question dépasse « le cas Wagner » et le problème de la contextualisation des représentations. Aucune musique n'est à l'abri d'une réappropriation que l'on pourrait juger abusive, que cela soit pour construire un rituel de mobilisation autour d'une marche ou que cela soit pour une quelconque publicité. Chostakovitch a été associé à une caisse de prévoyance... Dans le cas des marches, il est étonnant de voir leur plasticité dans des réemplois dans des contextes très différents (la Marche lorraine de Louis Ganne a pu, par exemple, être reprise par une milice libanaise). Il me semble difficile de distinguer, dans une approche historique, ce qui relève de l'emploi (plus ou moins ritualisé) et ce qui relève de ce que vous appelez les "significations ». On risque en parlant de signification d'une musique de retomber dans une forme d'essentialisme. A priori, en période de guerre, on fait feu de tout bois, on fond les statues pour forger des canons. Tout peut devenir arme de mobilisation. J'aurais tendance actuellement à choisir, au moins provisoirement, un parti pris théorique fonctionnaliste. La question se pose par exemple à propos de l'usage du folklore en Union Soviétique, y compris en temps de guerre. Des airs récents peuvent faire fonction de folklore dans un contexte donné. Les questions de ritualité n'interviennent pas seulement comme un signe d'une ritualité spécifique mais la ritualité "normale» du concert en temps de guerre est exploitée comme une arme de propagande. Parfois le fait d'exporter le rituel du concert 
hors des salles philharmoniques met en valeur, à des fins de propagande, la mobilisation confluente des artistes et du peuple. Furtwängler dirige en 1942 un concert aux usines A.E.G. de Berlin, avec d'ailleurs le Prélude des Maîtres Chanteurs. Il ne s'agit pas seulement de montrer que la vie normale continue et que l'ennemi n'a pas pu empêcher la nation de continuer à vivre : il s'agit de démontrer qu'elle est unanime et mobilisée. C'est en mars 1942 que l'Orchestre du Bolchoï, replié à Kouïbychev (aujourd'hui Samara), crée la VII e symphonie de Chostakovitch, reprise bien vite à Londres, à New York (sous la direction de Toscanini) et à Novossibirsk. Mais il faudrait également étudier des formes de ritualisation des musiques légères propres à la guerre : la tournée aux armées des vedettes, les photographies montrant les chanteurs ou les compositeurs en uniforme. En France, on connaît le cas des tournées de Maurice Chevalier ou de Joséphine Baker mais dans un pays comme la Finlande, la mobilisation des chanteurs et compositeurs de tango en Finlande (Olavi Virta, Henry Theel, Toïvo Kärki) sur le front de la Guerre d'Hiver et de la Guerre de Continuation a quelque chose de fondateur ou de refondateur de la nation. Une approche comparée, là encore, serait nécessaire.

LvP - Vous avez évoqué le rôle de la radio - en tant que média de masse - dans la diffusion et dans la fabrication des succès de la musique légère aussi bien dans les villes que sur les fronts. D'ailleurs, à en croire des témoignages laissés par des soldats après la Deuxième Guerre mondiale, le rituel quotidien de la radiodiffusion de certaines chansons aurait eu la capacité d'arrêter pour quelques minutes les hostilités entre belligérants - je pense notamment à la radiodiffusion de Lili Marleen par Radio Belgrade sur le front d'Afrique2. Ou, comme le met en scène le film Good Morning, Vietnam, le rôle de la radio a pu dépasser celui de la simple diffusion d'une programmation musicale visée par la censure militaire et revêtir une importance toute autre pour les troupes mobilisées. Pourriez-vous nous en dire un peu plus sur les fonctions de la radiodiffusion des œuvres musicales durant les conflits armés?

DF - La radio est, en période de guerre, un lien évident entre le front et l'arrière. En ce sens, les deux conflits mondiaux présentent des cas bien différents. La Seconde Guerre mondiale est largement une guerre des ondes. Le Discours du 18 juin, le brouillage de Radio Londres par les Allemands, tout cela constitue un paysage sonore identifié à l'histoire de cette guerre. Il est vrai que la musique légère n'est pas seule à occuper l'espace sonore radiophonique. Le signal radiophonique de Londres, le fameux $\mathrm{V}$ de la Victoire en morse, identifiable au début de la $\mathrm{V}^{\mathrm{e}}$ Symphonie de Beethoven peut difficilement passer pour de la musique «légère », pourtant le recours à la musique légère est fréquent, par exemple dans le retournement parodique avec Charlie et son orchestre avec lequel Goebbels pense démoraliser les auditeurs britanniques, ou avec le grand Pierre Dac se moquant de Radio Paris, station de collaboration, sur l'air de la Cucaracha avec des paroles restées célèbres («Radio Paris ment, Radio Paris ment, Radio Paris est allemand »). Si l'on étend la réflexion à la période de la Guerre Froide, le rôle de Radio Free Europe est considérable ; il serait intéressant de ne pas s'en tenir aux discours mais d'étudier précisément la programmation musicale, en comparant les émissions destinées aux différents pays du bloc soviétique. Je pense bien sûr à ce que vous dites sur Good Morning, Vietnam et donc à What a Wonderful World chanté par Armstrong mais largement pensé par Bob Thiele, le producteur des disques Impulse! Les œuvres musicales ayant ainsi apparemment une fonction de banalisation plus que de mobilisation.

LvP - Banalisation de la violence et de la guerre elle-même... Finalement, une guerre avec musique serait-elle moins pénible à vivre? Dans une perspective plus large, considérez- 
vous que la musique joue un rôle dans l'esthétisation de la violence guerrière vécue par les soldats?

DF - Je crois que ce sont deux questions bien différentes. D'un côté une sorte de vertu peut-être apaisante ou, au moins, d'euphémisation. Une forme illusoire de réhumanisation. Je pense, dans Le Dictateur, de Chaplin au moment où dans l'avion touché par l'artillerie et retombé sur terre, l'officier évoque la douceur du printemps dans son pays. La musique rend la guerre moins pénible à vivre. Il se peut qu'au $\mathrm{XX}^{\mathrm{e}}$ siècle, la fréquence des conflits ait joué un rôle dans la généralisation des musiques populaires qui ont pu avoir cette fonction: "on s'en sortira». L'amour, la séparation, les retrouvailles, des espèces de constantes « anthropologiques » qui font que la musique assure une forme de continuité fictive dans les destins par-delà les coupures, les déchirures et les blessures de la guerre. J'aime bien pour cela repérer la continuité en aval dans le souvenir nostalgique que laisse « la musique de la guerre». Je pense en particulier à une chanson de Charles Trénet, enregistrée, je pense, vers 1960 sur le souvenir de la Première Guerre mondiale, évoquant l'héroïne de la chanson emblématique de la période :

«Qu'est devenue, depuis,

La Madelon jolie

Des années seize?

A-t-elle toujours les yeux

Étonnés d'être si bleus,

La taille à l'aise?»

Le texte, nostalgique, est bien loin du pastiche et la musique n'évoque l'original que de très loin la musique de l'original et les musiques guerrières, sauf dans une introduction sur le mode des musiques d'ordonnance au clairon, qui s'estompe. La fin met en évidence le souvenir apaisant que laissent certaines musiques associées à l'histoire guerrière.

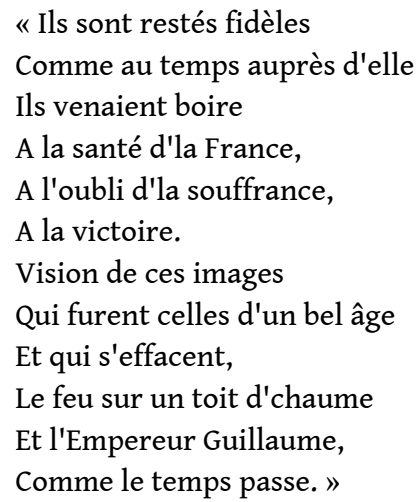

On retient presque de la guerre le souvenir de la chanson qui la rendait moins pénible. La question de l'esthétisation de la violence vécue par les combattants me semble une question bien différente. Si l'on cherche à réfléchir de façon générale en ne se limitant pas au front franco-allemand de la Première Guerre mondiale (dont la commémoration en France prend peut-être trop une allure uniquement franco-allemande, oubliant que la Guerre a touché aussi Sarajevo, Salonique ou Varsovie), on peut penser que l'esthétisation de la violence à laquelle la musique peut contribuer est avant tout l'affaire $d u$ "Front intérieur» de ce qu'avant l'idée de "guerre totale ${ }^{3}$ on appelait «l'arrière ». Les musiques produites par des musiciens qui ont eu l'expérience directe du combat semblent rarement belliqueuses. Là encore, la parodie est une forme non pas de dérision de l'adversaire mais de façon de maintenir une distance critique «civile » 
face au déferlement de la violence. Hindemith a ainsi parodié une forme de militarisme dans une vraie-fausse marche militaire pour quatuor à cordes «Militarminimax ». Ce qui me semble plus important que l'utilisation de la musique dans l'esthétisation de la violence est la capacité d'appropriation et de réappropriation de musiques en période de guerre, y compris au front. It's a Long Way to Tipperary est apparu au Royaume-Uni dans les music-halls en 1912 et s'est imposé comme air de marche des troupes britanniques à Boulogne-sur-Mer dans l'été 1914.

LvP - Pour terminer notre dialogue, je voudrais aborder le sujet des pratiques musicales au sein des armées en temps de guerre, dans une approche qui partirait du vécu des soldats eux-mêmes. De fait, si un certain nombre d'instruments de musique sont tolérés ou même mis à disposition dans les casernes pour un usage "récréatif », qu'en est-il des œuvres musicales ou des chansons que des soldats - musiciens amateurs ou professionnels auraient composées ou reprises au jour le jour, pour parodier la hiérarchie, pour ridiculiser l'ennemi, pour évoquer la nostalgie de leur vie loin du front, ou simplement pour passer du bon temps avec les camarades? A un niveau individuel, quelle fonction pouvaient avoir les pratiques musicales pour les soldats ou le personnel militaire? Quelles sources pourrionsnous mobiliser afın d'étudier ces témoignages du quotidien et de quelle façon une approche comparée pourrait-elle nous être utile?

DF - Beaucoup de choses déjà étudiées vont être utilement dites à l'occasion du Centenaire de la Première Guerre mondiale : du violon de Lucien Durosoir au répertoire étudié par Sophie-Anne Leterrier ${ }^{4}$. Je préfère essayer à présent de trouver d'autres exemples. Les compositions « au front » des musiciens finlandais dans la Guerre d'Hiver de 1939-1940 ou la Guerre de Continuation sont empreintes de l'« esprit de la Guerre d'Hiver » (Talvisodan Henki) qui est une forme d'Union Sacrée et donc n'expriment rien de critique face aux officiers supérieurs. Mais elles sont bien nostalgiques. Il est vrai que la diversité des conflits et des sources rend difficile tout essai de généralisation à partir de ce qui va être largement révélé ou rappelé à propos de la Grande Guerre. Pour cela, il y a de quoi reprendre avec Brassens l'idée qu'il s'agit bien de «celle que j'préfère ». On parvient, par les correspondances et les écrits autobiographiques, à avoir une certaine idée de ce qui est joué sur place, en particulier de ce qui est repris.

En définitive, il existe une forme assez codifiée de «folklore » militaire dans lequel on peut puiser : le répertoire des comiques troupiers en France, les danses de recrutement en Hongrie, les signaux d'ordonnance (l'appel à la soupe, par exemple), des chants de régiment sur lesquels il suffit de changer parfois légèrement les paroles. Une chanson polonaise consacrée aux "chevaux-légers» (Szwoleżerowie...) donne une idée de l'expression de cette « fraternité d'arme » musicale, avec parfois des allures de chanson à boire. Je crois qu'avec le processus de « folklorisation » du répertoire régimentaire, on peut construire une approche comparée ouverte des modalités d'appropriation des musiques jouées au front pendant les conflits. Vous avez raison d'insister dans vos questions sur le fait que la musique conserve une fonction dans la sociabilité («passer du bon temps avec les camarades »). La dimension comparée de l'étude de la place de la musique peut déjà s'appliquer dans l'étude historique de la Première Guerre mondiale. Je crains qu'en France on se concentre trop exclusivement sur les questions francoallemandes. Il faut relever autant que l'on peut les répertoires de tous les pays engagés dans le conflit, ce qui conduit à des choses aussi importantes que les premiers enregistrements de jazz avec James Europe, à d'indéboulonnables succès comme le Colonel Bogey associé, depuis un célèbre film, au Pont de la Rivière Kwaï, et donc à la Guerre mondiale suivante. C'est dire que le soutien à la recherche devrait faciliter les 
contacts internationaux pour rapprocher toutes les études partielles qui sont faites et leur donner un caractère systématique.

Même en période de conflit, le rapport affectif d'identification personnelle ou collective (par exemple nationale) à une certaine musique se fait à partir d'un répertoire largement mondialisé. Cela circule entre alliés. On a par exemple un enregistrement significatif d'une version anglaise de La Madelon. Mais cela circule aussi d'un camp à l'autre. Ce phénomène de construction de particularismes qui passe par la circulation transnationale est déjà bien présent avant 1914. Dans Le Chant des Nations, je m'étais prudemment arrêté à l'attentat de Sarajevo. Le fameux compositeur de marches tchèque Julius Fučík avait dirigé à Sarajevo la musique de garnison. Une de ses marches, L'Entrée des Gladiateurs, est devenue incontournable dans presque tous les cirques du monde. Pour les compositeurs patriotes, voire nationalistes, la reconnaissance passait par le succès dans des lieux internationaux de légitimation (Vienne, Paris ou Londres par exemple, voire même déjà New York où Gustav Mahler dirigea du Martucci). La construction du national est donc intimement liée à la circulation transnationale.

J'avais pensé en commençant Le Chant des Nations mettre en évidence la façon dont la musique a été instrumentalisée dans le processus de préparation des sociétés à la violence qui a abouti à la Première Guerre mondiale, j'ai plutôt trouvé des formes d'expression des nationalismes musicaux qui circulaient et se rapprochaient ainsi que des formes musicales de résistance au nationalisme. Il semble que l'étude des traductions effectuées dans le champ de l'édition et de la littérature européenne parvienne au même résultat alors que je pensais, en 2004, que la musique avait une sorte de facilité plus grande, ne passant pas par le truchement du traducteur, à concilier construction d'une culturelle nationale et transmission transnationale. Un article récent de Blaise Wilfert-Portal met en évidence les modalités, avant 1914, de «l'intensive construction du système transnational des littératures nationales européennes » en des termes en définitive assez proches, de façon surprenante, de ce que j'avais pu trouver à propos de la musique. Les « circulations » mettent en place à la fois des phénomènes de "nationalisation " et des phénomènes de «dépassement cosmopolite $»^{5}$. Et je trouve que le terme de " transnational » sur lequel il insiste à juste titre peut rendre compte de cette combinaison de mondialisation culturelle et de construction des particularismes nationaux que j'avais alors notée à propos de la musique. On ne peut que se réjouir de voir que, dans l'étude des phénomènes de circulation culturelle en Europe et hors d'Europe, les historiens qui travaillent sur la musique ne sont plus seuls à "dénationaliser » l'histoire culturelle. Il ne s'agit plus, et Blaise Wilfert-Portal insiste justement sur ce point, de mettre en place une méthode comparative mais de prendre comme objet ce que peuvent être «les bases collectives d'une histoire culturelle de l'Europe ». Pour ma part, je me consacre pour le moment essentiellement à l'observation de la circulation de musiques légères avant, pendant et après les guerres mondiales. Et cela circule dans des formes toujours étonnantes de géographie culturelle qui dépasse les frontières et les alliances diplomatiques et religieuses. Quelque chose se passe dès avant la Grande Guerre. Un exemple suffirait à le prouver. La valse d'Archibald Joyce, Songe d'Automne, qui fut peut-être la dernière œuvre jouée sur le Titanic, est devenue un immense succès avec des noms divers et des modifications en Suède, en Finlande, en Russie et en Serbie. Or cette valse triste (genre populaire de Sibelius au compositeur tchèque Oscar Nedbal) peut rappeler la valse russe 
sur Les Collines de Mandchourie qui évoque la fin héroïque des soldats morts lors de la Guerre Russo-Japonaise de 1904.

J'aurais presque envie de conclure en essayant d'exprimer l'idée que l'on peut préférer une démarche d'approche «trans » étudiant la circulation dans le cadre d'un large socle culturel commun plutôt qu'une démarche comparative «inter ». Par ailleurs, la fréquence des réemplois musicaux lors des guerres pourrait faire penser que dans le domaine musical, la fameuse formule de Clausewitz selon laquelle " La guerre n'est qu'un prolongement de la politique par d'autres moyens » pourrait s'appliquer. Les périodes de guerre apparaissent donc comme des moments où le phénomène de circulation est le plus paradoxal, le plus difficile, le plus contradictoire avec la logique d'affrontement et donc où on peut mieux mettre en évidence ses modalités «transgressives ». Il y a dans le «transnational» quelque chose de l'ordre de la transgression morale. Les réactions à ce qui est perçu comme l'américanisation (ou la mode wagnérienne, en d'autres temps) le montrent bien. L'étude de la circulation et de la traduction des textes pourra certainement apporter beaucoup à l'histoire d'une culture européenne, pour le moment il ne me semble pas que l'on ait encore épuisé le sujet des circulations musicales que je continue à privilégier. «De la musique avant toute chose » comme disait Verlaine qui n'hésitait pas à se présenter comme un poète patriotique français, "né à Metz ».

\section{BIBLIOGRAPHIE}

\section{Bibliographie sélective}

\section{Livre}

Le Chant des Nations. Musiques et Cultures en Europe, 1870-1914, Paris, Hachette, 2004.

\section{Directions d'ouvrages}

CLAVEAU, Cylvie, FISZER, Stanislaw et FRANCFORT, Didier (eds.), Cultures juives. Europe centrale et orientale, Amérique du Nord, Paris, Le Manuscrit, 2012, 545 p.

MASŁOWSKI, Michel, FRANCFORT, Didier et GRADVOHL, Paul (eds.), Culture et identité en Europe centrale. Canons littéraires et visions de l'histoire, Paris-Brno, Institut d'études slaves, 2011, 660 p.

DESHAYES, Jean-Luc et FRANCFORT, Didier (eds.), Du barbelé au pointillé : les frontières au regard des sciences humaines et sociales, Nancy, Presses Universitaires de Nancy, 2010, 297 p.

\section{Contributions à des ouvrages collectifs}

« La musique instrumentale du Risorgimento », in Laura FOURNIER-FINOCCHIARO et Jean-Yves FRÉTIGNÉ (eds.), L'Unité italienne racontée, vol. II : Voix et images du Risorgimento. Transalpina n 16, Caen, Presses universitaires de Caen, 2013, p. 167-179.

« Portrait de Frank Zappa en dissident tchèque : Circulations musicales est-ouest pendant la guerre froide ", in Musiques au monde. La tradition au prisme de la création, Emmanuelle OLIVIER (ed.), Sampzon, Delatour, 2012, p. 67-76. 
« Defining Musically the Enemy » in Nesrin KALYONCU, Derya ERICE et Metin AKYÜZ (eds.), Music and Music Education within the Context of Socio-cultural Changes, Ankara, Müzik Eğitimi Yayinlari, 2011, p. 224-228.

« La Guerre de Crimée, moment fondateur des musiques militaires européennes», in Georg MAAG, Wolfram PYTA, Martin WINDISCH (eds.), Der Krimkrieg als erster europäischer Medienkrieg. Berlin, LIT VERLAG, 2010, p. 163-172.

« Les musiques au cœur de la culture italienne » in Marc LAZAR (ed.), L'Italie contemporaine de 1945 à nos jours, Paris, Fayard, 2009, p. 429-440.

«La meilleure façon de marcher : musiques militaires, violence et mobilisation dans la Première Guerre mondiale », in La Grande Guerre des musiciens, Stéphane AUDOIN-ROUZEAU, Esteban BUCH, Myriam CHIMÈNES et Georgie DUROSOIR (eds.), Lyon, Symétrie, 2009, p. 17-27.

« National Identity and the Double Border in Lorraine 1870-1914 » in Barbara KELLY (ed.), French Music, Culture, and National Identity, 1870-1939, Rochester, New York, University of Rochester Press, 2008, p. 351-377.

\section{Articles}

«La Marseillaise de Serge Gainsbourg ", in Vingtième siècle. Revue d'histoire, nº 227, 2007/3, p. 27-35.

«Památník Lidicím, le "Mémorial de Lidice" de Bohuslav Martin 7 (1943) : réflexions sur l'engagement politique et moral d'un compositeur », in Guerres mondiales et conflits contemporains, $n^{\circ} 85,2005 / 1$, p. $65-74$.

«Pour une approche historique comparée des musiques militaires », in Vingtième siècle. Revue d'histoire, $\mathrm{n}^{\circ} 85,2005 / 1$, p. 85-101.

\section{NOTES}

1. FRANCFORT, Didier, Associations et pratiques sociables à Udine, métropole frioulane (1850-1870), 4 vol. (1074 p.), sous la direction de Maurice Agulhon, thèse soutenue à l'Université Paris-I le 7 mars 1986.

2. Voir à ce sujet SALA ROSE, Rosa, Lili Marleen. Canción de amor y de muerte, Barcelone, Global Rhythm, 2008, p. 109-113.

3. FRANCFORT, Didier, " Defining Musically the Enemy » in Nesrin KALYONCU, Derya ERICE et Metin AKYÜZ (eds.), Music and Music Education within the Context of Socio-cultural Changes, Ankara, 2011, p. 224-228.

4. https://www.google.fr/webhp?source=search_app\&gws_rd=cr\&ei=L1rSUv7CG-iu0AWhoGAAw\#q=sophie+anne+leterrier+musique+guerre [consulté le 12 janvier 2014]

5. WILFERT-PORTAL, Blaise, «L'histoire culturelle de l'Europe : un point de vue transnational » in Revista de Antropología e Arte, vol. 1, n. 4, 2012/2013, (http://www.revistaproa.com.br/04/? page_id=100), consulté le 12 janvier 2014. 


\section{RÉSUMÉS}

Professeur d'histoire contemporaine à l'Université de Lorraine et directeur de l'Institut d'Histoire Culturelle Européenne-Bronisław Geremek au Château des Lumières-Lunéville, Didier Francfort se considère comme un historien avec musique, et non pas un historien de la musique. Ses travaux portent sur l'histoire culturelle comparée européenne, particulièrement sur la place de la musique dans les constructions identitaires nationales ou supranationales. Il y analyse comment la musique intervient dans les sociétés à la fois comme source et comme facteur dans les évolutions sociales. Didier Francfort nous parle de son parcours d'historien et de la façon dont les œuvres musicales peuvent être mobilisées dans une approche historique transnationale et comparative. Dans la suite de l'entretien, il est question des œuvres et des pratiques musicales au sein des conflits armés, des deux guerres mondiales à celle du Vietnam.

\section{INDEX}

Mots-clés : histoire comparée, conflits armés, guerre, musique, pratiques musicales

Keywords : comparative history, armed conflicts, war, music, musical practices

\section{AUTEUR}

\section{LUIS VELASCO-PUFLEAU}

Docteur en Musique et musicologie (Université Paris-Sorbonne) et chercheur post-doctorant à l'Université de Salzbourg, Luis Velasco-Pufleau a été également chercheur post-doctorant au Centre de recherches sur les arts et le langage (CRAL) de l'École des hautes études en sciences sociales (EHESS, Paris) et chargé de cours en musicologie à l'Université de Bordeaux. Ses recherches portent sur la création musicale contemporaine ainsi que sur les rapports entre musique, esthétique et politique au $\mathrm{XX}^{\mathrm{e}}$ siècle. 